Published in final edited form as:

Cogn Affect Behav Neurosci. 2014 March ; 14(1): 90-105. doi:10.3758/s13415-013-0226-y.

\title{
The neural bases of distracter-resistant working memory
}

\author{
Tor D. Wager ${ }^{1,{ }^{*}}$, Julie Spicer ${ }^{2}$, Rachel Insler, and Edward E. Smith ${ }^{2,3,{ }^{* *}}$ \\ ${ }^{1}$ Department of Psychology and Neuroscience, University of Colorado, Boulder \\ 2Department of Psychology, Columbia University \\ ${ }^{3}$ Cognitive Neuroscience Division, New York State Psychiatric Institute
}

\begin{abstract}
A major difference between humans and other animals is our capacity to maintain information in working memory (WM) while performing secondary tasks, which enables sustained, complex cognition. A common assumption is that the lateral prefrontal cortex (PFC) is critical for WM performance in the presence of distracters, but direct evidence is scarce. We assessed the relationship between fMRI activity and WM performance within-subjects, with performance matched across Distracter and No-distracter conditions. Activity in ventrolateral PFC during WM encoding and maintenance positively predicted performance in both conditions, whereas activity in the pre-supplementary motor area (pre-SMA) predicted performance only under distraction. Other parts of dorsolateral and ventrolateral PFC predicted performance only in the No-distracter condition. These findings challenge a lateral PFC-centered view of distracter-resistance, and suggest that the lateral PFC supports a type of WM representation that is efficient for dealing with task-irrelevant input but is nonetheless easily disrupted by dual-task demands.
\end{abstract}

Working memory (WM) refers to a system that maintains currently relevant goals and information for use in guiding ongoing information processing. Information in WM is thought to guide the deployment of attention (de Fockert, Rees, Frith, \& Lavie, 2001) and the manipulation of information to achieve current task goals (Baddeley, 1986; Hitch \& Baddeley, 1976). Probably no factor has more effect on WM performance than the presence of distracters (e.g., Keppel \& Underwood, 1962). Consider the simple task of remembering a group of three letters - a trigram - over an interval of a few seconds. In a classic article (Peterson \& Peterson, 1959), participants rehearsed a trigram for intervals up to 18 seconds while counting backwards by threes. With this distracting secondary counting task, what would otherwise have been trivial to remember became virtually impossible, with near-zero recall by 18 seconds, with proactive inhibition amassed from prior trials having been shown to underlie this type of memory impairment (Keppel \& Underwood, 1962). The powerful effect of distraction is evident in everyday situations: Memory for the contents of a book or a phone number just memorized may be obliterated by a few moments of idle conversation.

Distracters in WM are of two basic types. They may not require any intentional processing, in which case they are simply perceptual experiences to be ignored while remembering other items, or they may constitute a secondary task that must be performed during maintenance of the memory set. Successful inhibition of merely perceptual distracters may rely on perceptual filtering mechanisms that can operate at an early processing stage (Bundesen, 1990; Treisman, 1985), whereas dual-task interference is likely to rely more heavily on task

\footnotetext{
"Corresponding author information: Tor D. Wager, Associate Professor of Psychology and Neuroscience, University of Colorado, Boulder, 345 UCB, Boulder, CO 80309, tor.wager@ colorado.edu, 303-492-7487.

*** Edward E. Smith (deceased August 17, 2012) contributed to this work through his close and invaluable involvement in study design, interpretation of results and manuscript preparation.
} 
switching, coordination, and information-selection processes. Performance on tasks involving these two distracter types is essentially uncorrelated (Miyake et al., 2000). Crucially, performance on WM tasks with dual-task distracters, rather than perceptual distracters, predicts performance on complex tests of fluid intelligence (Conway, Kane, \& Engle, 2003; Conway, Cowan, Bunting, Therriault, \& Minkoff, 2002; Engle, Kane, \& Tuholski, 1999; Fry \& Hale, 1996; Kane \& Engle, 2003; Kane \& Engle, 2002), correlates with other indices of 'executive function' (Bleckley, Durso, Crutchfield, Engle, \& Khanna, 2003; Kane \& Engle, 2003), and predicts everyday cognitive failures (Friedman \& Miyake, 2004).

Though there have been many studies investigating the brain mechanisms of selective attention and perceptual distracter suppression, very few studies have investigated those involved in creating WM representations resistant to dual-task distracters. The prefrontal cortex (PFC) is thought to be crucial for distracter resistance (Baddeley \& Della Sala, 1996; Knight, Richard Staines, Swick, \& Chao, 1999; Miller \& Cohen, 2001), but much of the key evidence has come from studies of perceptual distracters (Chao \& Knight, 1995, 1998; Jha, Fabian, \& Aguirre, 2004; Malmo, 1942; McNab \& Klingberg, 2008; Miller, Erickson, \& Desimone, 1996; Postle, 2005; Sreenivasan \& Jha, 2007; Tsushima, Sasaki, \& Watanabe, 2006). In an important study, Sakai, Rowe, \& Passingham (2002) examined the basis of resistance to dual-task distraction by assessing the relationship between PFC activity during WM encoding and maintenance with recall accuracy after performing a distracting secondary task. Activity during encoding/maintenance in right Brodmann's Area (BA) 46, which spans dorsolateral and ventrolateral prefrontal cortices (DLPFC/VLPFC), was greater for correct than error trials. On the basis of this finding, Sakai et al. (2002) suggested that this area is critical for distracter-resistant memory.

However, the Sakai et al. (2002) study was limited in a way that substantially undermines its conclusion. Though they showed an accuracy effect in the presence of a distracting task, they did not demonstrate that the accuracy effect was specific to the distraction condition. They did include a no-distraction condition, but participants made virtually no errors without distraction. Thus, the alternative remains that the lateral PFC plays a general role in WM maintenance with or without distraction. In addition, the accuracy probe on Sakai et al. (2002) WM task involved a binary "yes or no" recognition decision, precluding the possibility of assessing parametric relationships between brain activity and accuracy, which would help establish that there is a meaningful relationship between PFC activity and accuracy across the normal range of performance.

Two plausible alternatives for the role of the lateral PFC provide the basis for hypotheses in the current study. First, the lateral PFC may be important for WM maintenance with or without distracters. In several studies (D'Esposito \& Postle, 1999; Ptito, Crane, Leonard, Amsel, \& Caramanos, 1995), patients with lesions of Brodmann's Area 46 were impaired on delay tasks with or without distraction. Similarly, monkeys with prefrontal lesions have shown impaired performance on WM tasks with and without distracters (Funahashi, Bruce, \& Goldman-Rakic, 1989; Funahashi, Inoue, \& Kubota, 1997; Sawaguchi \& Yamane, 1999), though it has been argued that monkeys are distractible even without explicit distracters included in the task. Secondly, the lateral PFC may be particularly important for filtering out and reducing interference from perceptual distracters—as evidenced by neuroimaging studies (de Fockert et al., 2001; Dolcos, Miller, Kragel, Jha, \& McCarthy, 2007; Jha et al., 2004; Postle, 2005; Sreenivasan \& Jha, 2007), humans and monkeys with prefrontal damage (Chao \& Knight, 1995, 1998; Malmo, 1942), and primate electrophysiology (Artchakov et al., 2009; Miller et al., 1996; Tsushima et al., 2006)—but this region may not be crucial for resistance to dual-task distraction. This idea is consistent with evidence for a broad role for the VLPFC in particular in the selection of task-relevant sensory stimuli (Badre, Poldrack, 
Pare-Blagoev, Insler, \& Wagner, 2005; Fuster, 2008; Nee \& Jonides, 2009; ThompsonSchill et al., 2002). Thus, resisting perceptual distracters may load heavily on stimulus selection, whereas resisting dual-task distraction may load heavily on coordination and scheduling of WM processes, and thus place demands on different cortical areas.

We studied resistance to dual-task distraction using a standard verbal WM task with word memoranda and several novel features (Figure 1). First, like Sakai et al. (2002) we included both distracter and no-distracter conditions. In the distracter condition, there was a delay before onset of the secondary distracter task so that we could assess brain activity before distraction and relate it to subsequent accuracy. Second, to provide a parametric assessment of accuracy on each trial, we included a series of four yes/no recognition probes after each trial, resulting in five levels of accuracy ( $0-4$ correct). Finally, to permit a sensitive analysis of the brain-accuracy relationship for both distracter and no-distracter conditions, we used an adaptive staircase procedure to select a memory set size for each subject and each condition that resulted in approximately $75 \%$ accuracy ( $3 / 4$ probes correct on average) in both conditions. This allowed us to compare brain-accuracy relationships for distracter vs. no-distracter conditions, testing whether the lateral PFC and/or other areas show monotonic increases in activity with performance only under dual-task distracter conditions.

\section{Methods \\ Participants}

Seventeen healthy right-handed participants (mean age $=22.3$ years, 6 female) were recruited in compliance with the human subjects regulations of Columbia University and provided informed consent in accordance with the Declaration of Helsinki. They were paid US \$20/hour for voluntary participation, plus bonuses as described below. Handedness was assessed with the Edinburgh Handedness Inventory, and eligibility was assessed with a general health questionnaire and fMRI safety screening form.

\section{Materials and procedures}

Stimuli-Stimuli were selected from a list of two-syllable words ranging from four to six letters in length (mean 5.5), generated using the online version of the MRC Psycholinguistic Database (http://www.psy.uwa.edu.au/mrcdatabase/uwa_mrc.htm). Fifty nouns and fifty verbs were then each selected randomly from that list for use in the experiment. The words had intermediate average levels of concreteness $($ mean $=335)$, imageability ( and Kucera-Francis frequency $($ mean $=85)$. Words included in each trial were selected randomly with replacement from the list of 100 words. Items from this list could appear in both the encoding and the distraction events of the trial, with the constraint that items in the encoding event could not appear in the distraction event of the same trial. Due to the randomization procedure, items appeared approximately equally in the task, and a single trial included on average 50\% nouns and 50\% verbs. There were no restrictions on the number of nouns/verbs appearing in the encoding or distraction events.

Experimental structure-The experiment consisted of $80 \mathrm{WM}$ trials. As shown in Figure 1 , on each trial, participants intentionally encoded a series of words ("memory set"). The set size was chosen for each individual based on a pre-scan calibration (described below). During the encoding phase, each word was presented on a computer screen for $500 \mathrm{~ms}$ (with an approximate visual angle of 1 degree). Participants then maintained the word set over a $6000 \mathrm{~ms}$ maintenance period, during which a fixation cross was presented on the computer screen. How fMRI activity varied during these combined encoding and maintenance periods was of primary interest. 
Following maintenance, trials were divided into two types, which occurred with equal frequency. On distraction (D) trials, participants performed a secondary judgment task. They viewed a series of four words, each presented for $1200 \mathrm{~ms}$, and indicated whether each distracter was a noun or a verb by pressing the index or middle finger of the right hand, respectively, on an MRI-compatible button box. No words were members of the memory set. Participants were instructed to maintain the original memory set during the secondary task. A 2000 ms fixation period followed the set of distracters. On no-distraction (ND) trials, participants viewed a fixation cross for the same amount of total time as the distraction task (6800 ms) and continued to maintain the memory set.

$\mathrm{D}$ and ND trials were grouped in blocks of 10 trials, so that an entire scanning run consisted of either D or ND trials. Participants were told in advance that the trials would be blocked, and therefore had information that would allow them to prepare differently during encoding/ maintenance for D vs. ND trials.

Following the continued maintenance with or without the distracter task, participants viewed a series of 4 unique probe words, each presented for $1500 \mathrm{msec}$. Participants indicated whether each word was part of the memory set with a "yes" (index finger) or "no" (middle finger) response on the button box. This multistage probe period allowed for five levels of accuracy ( $0-4$ probes correct) on each trial, providing a more sensitive measure of WM performance than has previously been used. Probes were randomly selected from the memory set and from a set of words that was not part of the memory set ( 2 of each). Items were selected at random and were therefore on average approximately equally sampled. Sampling of items from the memory set was not stratified based on serial position of presentation. Although a serial rehearsal strategy could have been used by participants (as the items were likely rehearsed in some order, and using presentation order would be plausible), it would not confer a particular advantage at test. Following the probe period, a $20 \mathrm{sec}$ inter-trial interval allowed fMRI responses to return to approximate baseline values, which permitted us to quantify activity on individual trials.

Performance incentives-Participants were incentivized with instructions that they could receive a $\$ 0.10$ monetary reward on every trial for successful performance, with the potential to earn an additional $\$ 8.00$ over the course of the experiment. On ND trials "successful" performance for reinforcement purposes was defined solely in terms of memory performance, whereas D trials also required 3 or 4 correct responses to the 4 noun/ verb or yes/no judgments. Also, on D trials, participants received equivalent monetary incentives ( $\$ 0.05$ each) for good performance on both the probe and the distracting noun/ verb judgments to ensure that they would attend to both tasks. Participants did not receive any feedback about monetary earnings until the end of each block (scanning run). Bonuses ranged from $\$ 3.70$ to $\$ 5.30$, with an average of $\$ 4.61$.

Memory set titration-D and ND trials were matched for difficulty within and across participants during a titration phase that occurred before fMRI scanning. This titration phase also served to familiarize the participants with the 100 words used in the task to eliminate novelty effects. Participants performed the task using memory sets of varying sizes until they were achieving successful performance on $75 \%$ of the trials. The criterion of $75 \%$ correct ( 3 of 4 probes) was chosen because it lies midway between chance performance $(50 \%)$ and perfect accuracy $(100 \%)$, and thus maximizes power to detect accuracy effects by avoiding either ceiling or floor effects. Statistical power is maximized when there are equal numbers of trials at both ends of the useful range of an outcome (here, $2 / 4$ trials [chance] vs. $4 / 4$ trials [perfect]), as the predictor variance is maximal. 
The titration task was 40 trials long, with 1 block of D and ND trials each (20 trials per block). The task started with 8 words in the memory set of the D condition and 9 words in the memory set of the ND condition. After every 5 trials, set size updating occurred such that the size increased by 1 if accuracy exceeded $75 \%$ and decreased by 1 if accuracy fell below $75 \%$. After all trials, the final set size was determined by regressing trial-by-trial accuracy on trial-by-trial set size and then by using the regression line to identify the size associated with $75 \%$ correct.

Across participants and conditions, memory set size varied from 7 to 11 words. For each of the 17 participants, the titrated memory set size was larger in the ND condition (mean $=$ 10.77 words, s.d. $=0.56$ ) than in the D condition (mean $=9.24$ words, s.d. $=0.75$ ), indicating that the distracting noun/verb judgment task was having a deleterious effect on $\mathrm{WM}$ ability (mean difference $=1.53$ words, $\mathrm{t}(16)=12.25, \mathrm{p}<.001$ ). These set sizes were used in the subsequent fMRI task. Thus, the comparison in brain activity for [ND - D] trials differed in the number of words encoded, and might be expected to produce an overall [ND $>\mathrm{D}$ ] difference in standard WM networks (though if encoding/maintenance processes were more strongly engaged during D blocks, it would tend to offset the set size effect by creating a [D > ND] difference). However, the [ND - D] comparison was not of primary interest; we designed the study to focus on relationships between brain activity and accuracy within each condition. The titration ensured a homogenous distribution of accuracy values across participants and a distribution across a range of accuracy values within participants, for both the $\mathrm{D}$ and ND conditions, which is desirable for sensitive detection of brain-accuracy relationships.

\section{Data acquisition}

Whole-brain fMRI data were acquired on a 1.5T GE Signa Twin Speed Excite HD scanner (GE Medical Systems). Functional and anatomical images were acquired with a T2*sensitive EPI BOLD sequence with a TR of $2000 \mathrm{~ms}$, TE of $41 \mathrm{~ms}$, flip angle of $60^{\circ}$, ascending interleaved acquisition, field of view of $22 \mathrm{~cm}, 24$ slices and $3.44 \times 3.44 \times 4.5$ $\mathrm{mm}$ voxels, with whole-brain coverage. Stimulus presentation and data acquisition were controlled using Matlab (http://www.mathworks.com/) and the Psychophysics Toolbox (http://psychtoolbox.org/). An LCD projector displayed stimuli on a back-projection screen mounted in the scanner suite. Responses were made with the right hand via a 5 -finger button response unit with a molded hand brace (Resonance Technologies, Inc.).

\section{Image processing and data analysis}

Image denoising-Functional images were initially examined for spike artifacts using custom software (http://www.columbia.edu/cu/psychology/tor/). Global outlier time points were identified by computing both the mean and the standard deviation of values in each image for each slice. Mahalanobis distances for the matrix of mean values (one per slice) $\mathrm{x}$ functional volumes were computed, and images with a value above 3 standard deviations were considered outliers. Indicators for each individual outlier were entered as regressors in the general linear model (GLM). Next, principal components analysis was used to identify the first 10 components, and component scores were regressed on both outlier-related and task-related regressors (the design matrix; see below). Components that had strong outlierrelated correlations and weak task-related (e.g., r-squared values < .02) correlations were manually identified and removed from the data.

Preprocessing-After denoising, functional images were slice-acquisition timing and motion corrected using SPM5 software (Wellcome Department of Cognitive Neurology, UCL), using default parameters. Structural T1-weighted images were coregistered to the mean functional image for each subject using an iterative procedure of automated 
registration using mutual information coregistration in SPM5 and manual adjustment of the automated algorithm's starting point by a trained analyst until the automated procedure provided satisfactory alignment. Structural images were normalized (spatially warped) to a standard template brain (the MNI avg152T1.nii) SPM5's combined segmentation/ normalization procedure with default options, and the warping parameters were applied to functional images for each subject. Normalized functional images were interpolated to $2 \times 2$ $\times 2 \mathrm{~mm}$ voxels and spatially smoothed with an 8 -mm Gaussian filter.

First-level GLM model-First-level GLM analyses for each subject were estimated in SPM5. Quantification of single-trial response magnitudes was done by constructing a GLM design matrix with separate regressors for each trial, as in the "beta series" approach of Rissman et al. (Rissman, Gazzaley, \& D’Esposito, 2004). The model is a three-level mixed effects model, with trial nested within condition the first level, condition (D vs. ND x accuracy) within subjects at the second level, and subject as a random effect at the third level. For each $\sim 40 \mathrm{sec}$ trial, we included regressors for 1) the encoding/maintenance period, an 8.5 - $10.5 \mathrm{~s}$ epoch, depending on the number of words encoded; 2) the distraction/ maintenance period, a $6.8 \mathrm{~s}$ epoch; and the probe period, a $6 \mathrm{~s}$ epoch. As each trial was modeled individually, separate regression estimates were obtained for each D and ND trial. To assess activity during the encoding/maintenance period prior to the onset of distracters, and without assuming a response shape, we modeled epochs as boxcar functions (indicators) without convolving with a hemodynamic response function. All boxcars were delayed for 3 sec to compensate for the expected hemodynamic lag. An intercept regressor for each run modeled mean run activity.

The second-level analysis was conducted on first-level regression parameter estimates (activation estimates) for the encoding/maintenance period for each trial. To minimize the effects of MR artifacts on statistical results, trials with images coded as outliers or whose global brain signal values were greater than 3 s.d. from the mean were excluded (mean = 1.44 outliers per 100 images, s.d. $=0.51)$. For each voxel within each subject, a generalized least squares model was fit with an AR(1) error structure. Model predictors were Distracter Type (D vs. ND), Accuracy (an integer from 0 to 4), and the Distracter Type x Accuracy interaction (all predictors were centered). The intercept term in this model corresponded to the overall [Encoding/Maintenance vs. Baseline] contrast, where baseline was the $20 \mathrm{sec}$ inter-trial interval. These contrast images were subjected to group analysis (the third level of the model). A one-sample t-test across subjects (treating subject as a random effect) was performed for each of the four effects separately using robust regression, which increases statistical power and decreases false positive rates in the presence of outliers (Wager, Keller, Lacey, \& Jonides, 2005).

Search volume and localization of results-Normalized structural T1 images were averaged across participants to create an anatomical underlay for visualizing significant regions of activation, and for visually assessing normalization quality. In our experience, this is advantageous because the quality of nonlinear warping can vary across brain regions, resulting in greater differences between the standard brain and subjects' actual T1 images. The average T1 image was segmented using SPM5's unified segmentation/normalization routine, and the union of gray and white matter voxels was used as a mask in all analyses.

\section{Region of interest (ROI) analyses and multiple comparisons correction-We} defined ROIs based on the conjunction of three criteria: 1) gray/white matter, 2) functional response during the encoding/maintenance period, and 3) a priori interest based on previous studies. Our a priori hypotheses focused on two sets of regions. One set consisted of regions that were strongly activated in the overall [Encoding/Maintenance vs. Baseline] contrast (q $<.05$ false discovery rate (FDR) corrected, $\mathrm{p}<.0095$, see Figure 2 and Supplementary Table 
1) and were additionally located in lateral or medial PFC, which have been strongly implicated in cognitive control and resistance to distracters. A second set consisted of "default mode" regions (ventromedial PFC, posterior cingulate, superior temporal sulcus (STS)) and non-visual sensory processing regions (primary somatosensory cortex (S1) / secondary somatosensory cortex (SII), primary auditory cortex (AI)/SII) that are increasingly thought to play a role in task-irrelevant thought (Andrews-Hanna, Reidler, Huang, \& Buckner, 2010; Mason et al., 2007; McKiernan, D'Angelo, Kaufman, \& Binder, 2006), self-directed attention (Northoff et al., 2006), and poorer cognitive performance (Eichele et al., 2008; Weissman, Roberts, Visscher, \& Woldorff, 2006), and were also strongly deactivated in the [Encoding/Maintenance vs. Baseline] contrast $(\mathrm{q}<.05$ FDRcorrected). Within ROIs, we report results at a threshold of $\mathrm{p}<.001$, which is both the modal threshold used in fMRI studies and sufficient to control the family-wise error rate at $p$ $<.05$ across voxels within each ROI. However, for completeness and archival purposes, Supplementary Figure 1 (see Supplementary Material) shows results for all regions that met criteria (1) and (2) above.

\section{Results}

\section{Behavioral}

During the MRI session, mean accuracy was $71.21 \%($ s.e. $=1.81 \%$ ) in the ND condition and $61.91 \%$ (s.e. $=2.37 \%$ ) in the D condition. Mean d-prime was $1.30($ s.e. $=.14)$ in the ND condition and $.65($ s.e. $=.12)$ in the $\mathrm{D}$ condition. The results indicate overall that participants performed slightly below $75 \%$, the target level of accuracy determined by the memory set titration task and that they performed slightly better in the ND condition than the D condition.

\section{Encoding/Maintenance vs. Baseline}

Standard working-memory related regions were strongly activated during the encoding/ maintenance period (prior to distracter presentation; $\mathrm{p}<.01$ FDR). As shown in Figure 2A (hot colors, yellow/orange/red), activated regions included bilateral middle and inferior frontal gyri, intraparietal sulcus, inferior temporal cortices, pre-supplementary motor cortices (pre-SMA) and anterior cingulate (ACC). These results correspond closely with those from a recent meta-analysis of WM and related executive tasks (van Snellenberg \& Wager, 2009); see Figure 2B). Deactivation, shown in purple/blue in Figure 2A, was found in "default mode" regions associated with internal monitoring and task-unrelated thought during rest (Andrews-Hanna et al., 2010; Gusnard \& Raichle, 2001), including anterior medial prefrontal cortex (aMPFC), posterior cingulate (PCC), precuneus, inferior parietal cortex (IPC), and STS. In addition, deactivations were found in areas associated with sensory visual and somatosensory processing, including primary and secondary visual cortices (VI/VII), extrastriate cortex, dorsal posterior insula (dpINS) and SII. The combined activated and deactivated regions were used as a mask for subsequent analyses of accuracy.

\section{Predictors of subsequent accuracy}

We searched within a subset of functionally defined [Encoding/Maintenance - Baseline] ROIs of a priori interest (see Methods for region definition details) for subsequent accuracy effects. We expected activated areas in lateral and dorsomedial PFC to show positive accuracy-performance relationships-i.e., greater activity should predict parametric increases in subsequent accuracy - and deactivated "default mode" and somatosensory regions (dpINS and SII) to show negative accuracy-performance relationships.

We found monotonic relationships between brain activity increases and accuracy in three ROIs: the pars opercularis of the inferior frontal gyrus (IFG) bilaterally (Brodmann's Area 
44, based on the SPM Anatomy Toolbox v. 1.6 (Eickhoff et al., 2005), and the pre-SMA; both are shown in yellow in Figure 3. Detailed plots of responses for the IFG and pre-SMA are shown in Figures 4 and 5, respectively. Further analyses in all the areas that showed significant [Encoding/Maintenance - Baseline] responses revealed that there were additional regions besides these three that were positively correlated with accuracy (Figure 3; see Table 1 for statistics) including the premotor cortex, right anterior insula, inferior parietal lobule, left premotor cortex, and left inferior occipital/temporal cortex. These results are consistent with the hypothesis that increases in multiple regions with a distributed network support robust WM encoding and maintenance (cf. Pessoa, Gutierrez, Bandettini, \& Ungerleider, 2002).

Deactivation in several "default mode" and somatosensory ROIs also predicted subsequent WM accuracy (Table 1; negative relationships, shown in blue in Figure 3), including the pregenual cingulate/aMPFC, right STS, hippocampus, and bilateral IPC. All of these areas were deactivated during encoding/maintenance overall, and greater deactivation predicted increasing subsequent WM accuracy. In addition, right SII showed the same pattern of stronger deactivation with increasing accuracy. In the broader search within all [Encoding/ Maintenance - Baseline] areas, negative relationships with accuracy were also found in lateral occipital cortex and several motor/premotor regions. These findings are broadly consistent with the idea that accurate WM encoding and maintenance rely on reduced activation in brain systems that encode task-unrelated perceptual and motor processes (e.g., Andrews-Hanna et al., 2010).

\section{Predictors of distracter-resistant memory}

Regions in which activation during encoding/maintenance supports distracter-resistant memory should (1) be activated during encoding/maintenance, (2) positively predict accuracy overall, and (3) show a positive interaction with Distraction, indicating a stronger accuracy effect on D trials relative to ND trials. Thus, to assess regions that differentially predicted accuracy under D and ND conditions, we tested the Distraction x Accuracy interaction in the average signal within accuracy-predictive regions discussed above (i.e., regions listed in Table 1).

Only one region, pre-SMA, fit all of the criteria (Figure 4). Positive Accuracy and Accuracy $x$ Distracter effects indicated that activity was more positively related to subsequent memory for D than ND trials ( $p<0.05$; see Table 1). Furthermore, post hoc tests revealed that the accuracy effect was significant for D trials $(\mathrm{t}(16)=2.86, \mathrm{p}=0.01$ ) but not for ND trials $(\mathrm{t}(16)=-.33, \mathrm{p}=0.74)$, suggesting that pre-SMA activity is important for establishing distracter-resistant memory. In contrast, the bilateral IFG regions that predicted accuracy were equally predictive for D and ND trials, as shown in Figure 5 (see Table 1 for statistics).

It is possible that distracter-resistant memories are encoded in regions of the PFC other than our stringently defined a priori ROIs, which required significant [Encoding/Maintenance Baseline] activity and positive accuracy prediction across all trials. To test this possibility, we searched for voxels that showed Accuracy x Distracter effects at a threshold of $p<.001$ across the frontal cortex. The results revealed both positive and negative Accuracy $x$ Distracter interactions. Only one region of right middle frontal gyrus (MFG; BA 9/46; orange in Figure 6) showed a positive relationship with performance only in the D condition, as predicted by the distracter-resistance account of lateral prefrontal function. It showed [Encoding/Maintenance - Baseline] activation overall, but at a lower threshold $(\mathrm{t}(16)=3.04$, $\mathrm{p}=.008)$, and was not predictive of accuracy across all trial types. Other prefrontal regions, including bilateral MFG (BA 45), premotor cortex (BA 6) and left VLPFC (BA 47/12), showed a negative interaction (blue in Figure 6). Examining the pattern of these interactions revealed that in right premotor and VLPFC, the interaction was driven mainly by an 
association between greater activity and lower levels of accuracy on D trials, contrary to the distracter-resistance account of lateral PFC function. In MFG (BA 45), the interaction was driven mainly by stronger positive brain-accuracy relationships on ND trials. These findings are inconsistent with the idea that lateral PFC facilitates distracter-resistant WM.

In addition to activated areas in which the magnitude of increases predicts subsequent memory, we expected that the magnitude of decreases in deactivated areas may predict subsequent memory specifically on D trials. Results for "default mode" regions of interest are shown in Figure 7. Among areas in which deactivation magnitude predicted accuracy across all trials (Figure 3 and Figure 7A, left), three regions showed interactions with distracter status (Figure 7A, right): Right SII and dpINS, associated with somatic sensory perception, and STS, broadly associated with spontaneous cognition and social cognition (see Discussion). However, the Distracter $x$ Accuracy interaction in each of these areas was positive, indicating that decreases in activity were most beneficial for accurate performance in the ND condition. For example, as shown in Figure 7B, low accuracy in the ND condition in particular was associated with increased SII activity. These results underscore the idea that different processes may be rate-limiting steps in performance with and without distraction.

In contrast, aMPFC, a part of the "default mode" network, showed a trend toward stronger deactivation with more accurate memory specifically on D trials. Though this effect did not reach significance for the Accuracy $x$ Distracter interaction in the ROI defined by overall subsequent accuracy effects (Table 1), an adjacent region just outside the accuracypredictive search mask did show an Accuracy $x$ Distracter effect at $\mathrm{p}<.001(\mathrm{xyz}=[6,58$, 8], 2 voxels, $\max t=9.50$; shown in Figure 7C). Thus, deactivation of aMPFC may also be important for creating distracter-resistant memory.

\section{Discussion}

In the present study, we assessed the role of PFC and other regions in creating memories resistant to dual-task distracters by using a WM paradigm with two novel elements. First, we matched performance on D and ND versions of the task by adaptively titrating the memory set size, ensuring that we could efficiently estimate brain activity-performance relationships for both tasks. Second, we used a multi-part recognition probe with five levels of accuracy per trial, enhancing our ability to assess activity-performance relationships within-subjects. This approach is relatively unique, because in contrast to the hundreds of published WM studies, only a handful have reported relationships between PFC activity and behavioral performance (Gray, Chabris, \& Braver, 2003; Jha et al., 2004; Pessoa et al., 2002; Rypma, Berger, \& D'Esposito, 2002; Rypma \& D'Esposito, 2003; Smith et al., 2001).

We replicated earlier results of Sakai et al. (2002) by showing that activation during encoding/maintenance in both the lateral and medial PFC predicted successful subsequent performance under distraction. However, among rigorously defined functional ROIs, only activation in the pre-SMA predicted performance specifically under distraction. Activity in VLPFC predicted subsequent performance in both $\mathrm{D}$ and ND conditions of the task, and was not selective to the D task. An exploratory search outside of the strict boundaries of our functional localizers also revealed an area in the right DLPFC (BA 46) close to Sakai et al.'s (2002) results in which activation showed a more positive relationship with subsequent performance under D than ND conditions. However, activation of nearby and more widespread areas within bilateral IFG and DLPFC (bilateral BA 45 and left BA 47) predicted accurate performance only in the ND task, in opposition to the distracter-resistance hypothesis of lateral PFC function. 
The present results suggest that pre-SMA, which has been activated in numerous WM tasks (Rottschy et al., 2012) but has received less attention in studies of WM than lateral PFC, plays a more important role than previously thought in maintaining representations that are resistant to dual-task interference, which is particularly important for complex cognition. Pre-SMA was a) activated during WM encoding/maintenance; b) correlated positively with subsequent WM accuracy across all trials on average; and c) correlated more positively for D than ND conditions. Furthermore, the activation-performance correlation appeared to be exclusively driven by the $\mathrm{D}$ condition. This finding is consistent with a recent meta-analysis of executive function in working memory linking distracter resistance to pre-SMA (Nee et al., 2013) and is interesting because there is substantial evidence that pre-SMA plays a very general role in goal-directed cognition and action-selection across many tasks (Hampshire, Duncan, \& Owen, 2007; Johnson et al., 2005; Passingham, Chen, \& Thaler, 1989; Raye, Mitchell, Reeder, Greene, \& Johnson, 2008; Shima \& Tanji, 1998; Stuss \& Alexander, 2007; van Snellenberg \& Wager, 2009) including preparation (Brass \& von Cramon, 2002, 2004; Chikazoe et al., 2009). Thus, it may be the strength of engagement of basic representation-selection and rehearsal processes that create distracter-resistant $\mathrm{WM}$, rather than a circuit dedicated specifically to managing distraction.

Our results also have implications for the role of lateral PFC in WM, and suggest the somewhat surprising conclusion that in contrast to the dominant view (Kane \& Engle, 2002; Miller \& Cohen, 2001), lateral PFC may play a limited role in managing dual-task situations in healthy individuals. The fact that multiple areas in VLPFC and DLPFC, along with temporal regions, predicted performance more strongly in the ND condition implies that memory-set representations in prefrontal-temporal circuits are fragile and are disrupted by distracters. Performance correlations identify areas that implement 'performance-limiting' processes, or processes that fail intermittently and thus influence accuracy. VLPFC and DLPFC are almost certainly involved in task representation and maintenance, but the critical determinant of accuracy under dual-task distraction may be another facet of the representation-e.g., semantic or multi-modal associations that allow the memory to persist. The creation of a more fragile memory representation in the ND condition is also consistent with the idea that activation of task-irrelevant representations (e.g., presumably somatosensory-related activity in S2) might be more disruptive in the ND condition, which was the case here. Whether VLPFC supports an efficient but fragile type of encoding remains to be investigated in future experiments.

This conception of lateral PFC function is not inconsistent with previous work on distraction in WM, provided that the distinction between perceptual distracters and dual-task distraction is acknowledged. For instance, Chao and Knight (Chao \& Knight, 1998; Chao, NielsenBohlman, \& Knight, 1995) found that patients with lateral PFC damage showed impaired WM performance with perceptual distracters (auditory tones) and increased electrophysiological responses to such tones, suggesting reduced distracter inhibition. In monkeys, Miller et al. (1996) reported that activity in prefrontal neurons persisted across a distracter-filled delay period, but activity in inferior temporal neurons was disrupted by distracter presentation. In humans, Jha et al. (2004) reported greater lateral PFC activity during WM maintenance with concurrent presentation of perceptual distracters that were similar to the memory items. These findings and others support the idea that LPFC is important for stimulus selection and reduction of proactive interference (Badre \& Wagner, 2005; Feredoes, Tononi, \& Postle, 2006; Fuster, 2008; Nee \& Jonides, 2009; Nelson, Reuter-Lorenz, Sylvester, Jonides, \& Smith, 2003; Thompson-Schill, D’Esposito, Aguirre, $\&$ Farah, 1997), i.e., keeping irrelevant representations out of mind, but not management of concurrent task sets required for resistance to dual-task distraction. As very few studies have examined the neural bases of dual-task distraction (for discussion see Chein, Moore, \& Conway, 2011), and management of dual-task distraction is particularly predictive of 
complex cognitive abilities (Kane et al., 2004; Miyake et al., 2000), the present results serve as a launching point for explorations of the brain systems most critical for enabling complex cognition.

Here, another set of findings that argues against a lateral-PFC-dominated view of WM maintenance is the robust relationship between WM performance and deactivation of "default mode" and sensory processing regions. "Default mode" regions are associated with mentalization about the self (Northoff et al., 2006), autobiographical memory retrieval (McDermott, Szpunar, \& Christ, 2009), affective arousal (Wager et al., 2008), emotion (Wager et al., 2008), and task-irrelevant thoughts (Andrews-Hanna et al., 2010; Christoff, Gordon, Smallwood, Smith, \& Schooler, 2009; Mason et al., 2007; Wang et al., 2009). These findings parallel similar relationships between "default mode" decreases and performance on basic attention tasks (Eichele et al., 2008; Weissman et al., 2006) and longterm memory encoding (Daselaar et al., 2009).

Thus, though lateral PFC increases may be a robust feature of WM processes, and an intact PFC may be critical for complex memory operations, the lateral PFC may be "necessary but not sufficient" for good performance in the context of dual-task distraction. In contrast, activation in pre-SMA (likely related to response-generation) and deactivation of regions involved in spontaneous, internally generated cognition may be critical.

\section{Limitations and methodological considerations}

This study was designed to assess brain-performance relationships for distracting vs. nondistracting WM conditions, and so required that performance be matched in the two conditions, and that the task be difficult enough to be in a sensitive range for brainperformance correlations for each subject. To do this, we opted to titrate the memory set size for each subject for each task, resulting in a higher memory-set size for the ND than the D condition. Because of this, we did not attempt to compare the main effects of the D vs. ND conditions on brain activity. Previous studies have supplied this information, reporting increased activity in DLPFC (Jha et al., 2004; Postle, 2005), MFG (McNab \& Klingberg, 2008) and VLPFC (Jha et al., 2004), for example, during processing of task-relevant distracters.

Additionally, we did not opt to titrate the number of distracters. As stated above, we varied the size of the memory set in order to match performance on $\mathrm{D}$ and ND trials and provide a relatively highly-powered assessment of D vs. ND x accuracy effects. We did not titrate the number of distracters as doing so would have introduced variance across individuals in the working memory delay period, which we believed would introduce additional unwanted complexity in the design, as the delay duration substantially impacts working memory performance. Thus, we cannot ensure that the distracters were equally distracting for all participants, but this did not affect our ability to conduct the within-subject contrasts (accuracy, D vs. ND x accuracy) of primary interest. It might have been possible to design a titrated-distracter version of the task, but this was not mandated by our hypotheses.

Another design choice was to block the $\mathrm{D}$ and ND conditions, thus preventing confusion and avoiding task switching on the part of participants. Because participants knew whether the distracters would appear or not, they could easily adopt different strategies (based possibly on differing expectancies and/or preparation processes) in D vs. ND conditions. Thus, we cannot tell whether it is the type of representation or the type of strategy employed that causes different regions to predict performance in each case. However, we regard this as an important and ecologically valid feature of the task: Knowing that one will be distracted affords the opportunity to engage in robust encoding and maintenance processes, strategic or otherwise. Such knowledge is a feature of complex WM span tasks that predict general fluid 
intelligence. Future studies could specifically investigate the impact of knowledge about upcoming distraction.

Fourth, it might be argued that the high WM load in our task produced "distraction" in the D and ND conditions alike. However, the double dissociations in the brain-performance correlations in $\mathrm{D}$ and ND conditions suggest that these two conditions are not equally distracting, or at least not in the same ways. As we argued above, interference due to memory load should be thought of as conceptually distinct from the requirement to manage a demanding secondary task.

Fifth, it might be argued that ours was not a WM task given that we presented up to 11 twosyllable words, which may go beyond what is considered WM capacity, and given that we used probes to test memory at the end of each trial, raising the possibility that high performance was achieved by means of long term memory or non-WM strategies. In light of these potential concerns, we note the following ideas: 1) the task used a standard delay and probe for WM based on Sternberg's classic work; 2) the task elicited activation in standard WM brain areas; 3 ) potential chunking of verbal material in WM is consistent with capacity limits; and 4) the task was not designed to excise long term memory processes - indeed, long term memory processes have been implicated in WM in many situations. Thus, we believe this task was one that tested WM.

Sixth, we did not attempt to separate encoding and maintenance processes, because doing so would have come at a substantial cost in power for our main fMRI comparisons. Future studies might fruitfully separate these processes.

Finally, the task we used was designed to study the mechanisms of WM maintenance, but is not isomorphic with the tasks and task parameters shown to predict fluid intelligence.

Studying such tasks with fMRI (Chein et al., 2011) and correlating fMRI activity with fluid intelligence directly (Gray et al., 2003) is an important future direction.

\section{Supplementary Material}

Refer to Web version on PubMed Central for supplementary material.

\section{Acknowledgments}

We would like to thank Matthew Davidson for assistance with data analysis and manuscript preparation. This work was supported in part by grants NSF 0631637 and R01MH076136 (T.D.W).

\section{References}

Andrews-Hanna JR, Reidler JS, Huang C, Buckner RL. Evidence for the default network's role in spontaneous cognition. J Neurophysiol. 2010; 104(1):322-335.10.1152/jn.00830.2009 [PubMed: 20463201]

Artchakov D, Tikhonravov D, Ma Y, Neuvonen T, Linnankoski I, Carlson S. Distracters impair and create working memory-related neuronal activity in the prefrontal cortex. Cereb Cortex. 2009; 19(11):2680-2689.10.1093/cercor/bhp037 [PubMed: 19329569]

Baddeley, A. Working Memory. New York: Oxford University Press; 1986.

Baddeley A, Della Sala S. Working memory and executive control. Philosophical Transactions of the Royal Society of London - Series B: Biological Sciences. 1996; 351(1346):1397-1403. discussion 1403-1394.

Badre D, Poldrack RA, Pare-Blagoev EJ, Insler RZ, Wagner AD. Dissociable controlled retrieval and generalized selection mechanisms in ventrolateral prefrontal cortex. Neuron. 2005; 47(6):907918.10.1016/j.neuron.2005.07.023 [PubMed: 16157284] 
Badre D, Wagner AD. Frontal lobe mechanisms that resolve proactive interference. Cereb Cortex. 2005; 15(12):2003-2012.10.1093/cercor/bhi075 [PubMed: 15788702]

Bleckley MK, Durso FT, Crutchfield JM, Engle RW, Khanna MM. Individual differences in working memory capacity predict visual attention allocation. Psychon Bull Rev. 2003; 10(4):884-889. [PubMed: 15000535]

Brass M, von Cramon DY. The role of the frontal cortex in task preparation. Cereb Cortex. 2002; 12(9):908-914. [PubMed: 12183390]

Brass M, von Cramon DY. Decomposing components of task preparation with functional magnetic resonance imaging. J Cogn Neurosci. 2004; 16(4):609-620.10.1162/089892904323057335 [PubMed: 15165351]

Bundesen C. A theory of visual attention. Psychological review. 1990; 97(4):523-547. [PubMed: 2247540]

Chao LL, Knight RT. Human prefrontal lesions increase distractibility to irrelevant sensory inputs. Neuroreport. 1995; 6(12):1605-1610. [PubMed: 8527724]

Chao LL, Knight RT. Contribution of human prefrontal cortex to delay performance. J Cogn Neurosci. 1998; 10(2):167-177. [PubMed: 9555105]

Chao LL, Nielsen-Bohlman L, Knight RT. Auditory event-related potentials dissociate early and late memory processes. Electroencephalogr Clin Neurophysiol. 1995; 96(2):157-168. [PubMed: 7535221]

Chein JM, Moore AB, Conway AR. Domain-general mechanisms of complex working memory span. Neuroimage. 2011; 54(1):550-559.10.1016/j.neuroimage.2010.07.067 [PubMed: 20691275]

Chikazoe J, Jimura K, Hirose S, Yamashita K, Miyashita Y, Konishi S. Preparation to inhibit a response complements response inhibition during performance of a stop-signal task. J Neurosci. 2009; 29(50):15870-15877.10.1523/JNEUROSCI.3645-09.2009 [PubMed: 20016103]

Christoff K, Gordon AM, Smallwood J, Smith R, Schooler JW. Experience sampling during fMRI reveals default network and executive system contributions to mind wandering. Proc Natl Acad Sci U S A. 2009; 106(21):8719-8724.10.1073/pnas.0900234106 [PubMed: 19433790]

Conway AR, Kane MJ, Engle RW. Working memory capacity and its relation to general intelligence. Trends Cogn Sci. 2003; 7(12):547-552. S1364661303002833. [PubMed: 14643371]

Conway ARA, Cowan N, Bunting MF, Therriault DJ, Minkoff SRB. A latent variable analysis of working memory capacity, short-term memory capacity, processing speed, and general fluid intelligence. Intelligence. 2002; 30(2):163-183.

D'Esposito M, Postle BR. The dependence of span and delayed-response performance on prefrontal cortex. Neuropsychologia. 1999; 37(11):1303-1315. S0028393299000214. [PubMed: 10530730]

Daselaar SM, Prince SE, Dennis NA, Hayes SM, Kim H, Cabeza R. Posterior midline and ventral parietal activity is associated with retrieval success and encoding failure. Front Hum Neurosci. 2009; 3:13.10.3389/neuro.09.013.2009 [PubMed: 19680466]

de Fockert JW, Rees G, Frith CD, Lavie N. The role of working memory in visual selective attention. Science. 2001; 291(5509):1803-1806.10.1126/science.1056496291/5509/1803 [PubMed: 11230699]

Dolcos F, Miller B, Kragel P, Jha A, McCarthy G. Regional brain differences in the effect of distraction during the delay interval of a working memory task. Brain Res. 2007; 1152:171181.10.1016/j.brainres.2007.03.059 [PubMed: 17459348]

Eichele T, Debener S, Calhoun VD, Specht K, Engel AK, Hugdahl K, Ullsperger M. Prediction of human errors by maladaptive changes in event-related brain networks. Proc Natl Acad Sci U S A. 2008; 105(16):6173-6178.10.1073/pnas.0708965105 [PubMed: 18427123]

Eickhoff SB, Stephan KE, Mohlberg H, Grefkes C, Fink GR, Amunts K, Zilles K. A new SPM toolbox for combining probabilistic cytoarchitectonic maps and functional imaging data. Neuroimage. 2005; 25(4):1325-1335.10.1016/j.neuroimage.2004.12.034 [PubMed: 15850749]

Engle RW, Kane MJ, Tuholski SW. Individual differences in working memory capacity and what they tell us about controlled attention, general fluid intelligence, and functions of the prefrontal cortex. Models of working memory: Mechanisms of active maintenance and executive control. 1999:102134. 
Feredoes E, Tononi G, Postle BR. Direct evidence for a prefrontal contribution to the control of proactive interference in verbal working memory. Proc Natl Acad Sci U S A. 2006; 103(51): 19530-19534.10.1073/pnas.0604509103 [PubMed: 17151200]

Friedman NP, Miyake A. The relations among inhibition and interference control functions: A latentvariable analysis. Journal of Experimental Psychology-General. 2004; 133(1):101. [PubMed: 14979754]

Fry AF, Hale S. Processing speed, working memory, and fluid intelligence: Evidence for a developmental cascade. Psychological science. 1996; 7(4):237.

Funahashi S, Bruce CJ, Goldman-Rakic PS. Mnemonic coding of visual space in the monkey's dorsolateral prefrontal cortex. J Neurophysiol. 1989; 61(2):331-349. [PubMed: 2918358]

Funahashi S, Inoue M, Kubota K. Delay-period activity in the primate prefrontal cortex encoding multiple spatial positions and their order of presentation. Behav Brain Res. 1997; 84(1-2):203223. [PubMed: 9079786]

Fuster, JM. The prefrontal cortex. 4. London: Academic Press; 2008.

Gray JR, Chabris CF, Braver TS. Neural mechanisms of general fluid intelligence. Nat Neurosci. 2003; 6(3):316-322.10.1038/nn1014 [PubMed: 12592404]

Gusnard DA, Raichle ME. Searching for a baseline: functional imaging and the resting human brain. Nat Rev Neurosci. 2001; 2(10):685-694.10.1038/35094500 [PubMed: 11584306]

Hampshire A, Duncan J, Owen AM. Selective tuning of the blood oxygenation level-dependent response during simple target detection dissociates human frontoparietal subregions. J Neurosci. 2007; 27(23):6219-6223. 27/23/6219. 10.1523/JNEUROSCI.0851-07.2007 [PubMed: 17553994]

Hitch GJ, Baddeley AD. Verbal reasoning and working memory. The Quarterly Journal of Experimental Psychology. 1976; 28(4):603-621.

Jha AP, Fabian SA, Aguirre GK. The role of prefrontal cortex in resolving distractor interference. Cogn Affect Behav Neurosci. 2004; 4(4):517-527. [PubMed: 15849894]

Johnson MK, Raye CL, Mitchell KJ, Greene EJ, Cunningham WA, Sanislow CA. Using fMRI to investigate a component process of reflection: prefrontal correlates of refreshing a just-activated representation. Cogn Affect Behav Neurosci. 2005; 5(3):339-361. [PubMed: 16396094]

Kane MJ, Engle RW. Working-memory capacity and the control of attention: the contributions of goal neglect, response competition, and task set to Stroop interference. J Exp Psychol Gen. 2003; 132(1):47-70. [PubMed: 12656297]

Kane MJ, Hambrick DZ, Tuholski SW, Wilhelm O, Payne TW, Engle RW. The generality of working memory capacity: a latent-variable approach to verbal and visuospatial memory span and reasoning. J Exp Psychol Gen. 2004; 133(2):189-217.10.1037/0096-3445.133.2.189 [PubMed: 15149250]

Kane MJ, Engle RW. The role of prefrontal cortex in working-memory capacity, executive attention, and general fluid intelligence: An individual-differences perspective. Psychonomic Bulletin \& Review. 2002; 9(4):637. [PubMed: 12613671]

Keppel G, Underwood BJ. Proactive inhibition in short-term retention of single items. Journal of Verbal Learning and Verbal Behavior. 1962; 1(3):153-161.

Knight RT, Richard Staines W, Swick D, Chao LL. Prefrontal cortex regulates inhibition and excitation in distributed neural networks. Acta Psychologica. 1999; 101(2-3):159-178. [PubMed: 10344184]

Malmo RB. Interference factors in delayed response in monkeys after removal of frontal lobes. Journal of Neurophysiology. 1942; 5(4):295.

Mason MF, Norton MI, Van Horn JD, Wegner DM, Grafton ST, Macrae CN. Wandering minds: the default network and stimulus-independent thought. Science. 2007; 315(5810):393-395.10.1126/ science.1131295 [PubMed: 17234951]

McDermott KB, Szpunar KK, Christ SE. Laboratory-based and autobiographical retrieval tasks differ substantially in their neural substrates. Neuropsychologia. 2009; 47(11):2290-2298.10.1016/ j.neuropsychologia.2008.12.025 [PubMed: 19159634]

McKiernan KA, D'Angelo BR, Kaufman JN, Binder JR. Interrupting the "stream of consciousness": an fMRI investigation. Neuroimage. 2006; 29(4):1185-1191.10.1016/j.neuroimage.2005.09.030 [PubMed: 16269249] 
McNab F, Klingberg T. Prefrontal cortex and basal ganglia control access to working memory. Nat Neurosci. 2008; 11(1):103-107.10.1038/nn2024 [PubMed: 18066057]

Miller EK, Cohen JD. An integrative theory of prefrontal cortex function. Annu Rev Neurosci. 2001; 24:167-202.10.1146/annurev.neuro.24.1.167 [PubMed: 11283309]

Miller EK, Erickson CA, Desimone R. Neural mechanisms of visual working memory in prefrontal cortex of the macaque. J Neurosci. 1996; 16:5154-5167. [PubMed: 8756444]

Miyake A, Friedman NP, Emerson MJ, Witzki AH, Howerter A, Wager TD. The unity and diversity of executive functions and their contributions to complex "Frontal Lobe" tasks: a latent variable analysis. Cognit Psychol. 2000; 41(1):49-100. [PubMed: 10945922]

Nee DE, Brown JW, Askren MK, Berman MG, Demiralp E, Krawitz A, Jonides J. A meta-analysis of executive components of working memory. Cereb Cortex. 2013; 23(2):264-282.10.1093/cercor/ bhs007 [PubMed: 22314046]

Nee DE, Jonides J. Common and distinct neural correlates of perceptual and memorial selection. Neuroimage. 2009; 45(3):963-975. [PubMed: 19280708]

Nelson JK, Reuter-Lorenz PA, Sylvester CY, Jonides J, Smith EE. Dissociable neural mechanisms underlying response-based and familiarity-based conflict in working memory. Proc Natl Acad Sci U S A. 2003; 100(19):11171-11175.10.1073/pnas.1334125100 [PubMed: 12958206]

Northoff G, Heinzel A, de Greck M, Bermpohl F, Dobrowolny H, Panksepp J. Self-referential processing in our brain--a meta-analysis of imaging studies on the self. Neuroimage. 2006; 31(1): 440-457.10.1016/j.neuroimage.2005.12.002 [PubMed: 16466680]

Passingham, RE.; Chen, YC.; Thaler, D. Supplementary motor cortex and self-initiated movement. In: Ito, M., editor. Neural Programming. Basel: S. Karger AG; 1989. p. 13-24.

Pessoa L, Gutierrez E, Bandettini P, Ungerleider L. Neural correlates of visual working memory: fMRI amplitude predicts task performance. Neuron. 2002; 35(5):975-987. S0896627302008176. [PubMed: 12372290]

Peterson LR, Peterson MJ. Short-term retention of individual verbal items. J Exp Psychol. 1959; 58:193-198. [PubMed: 14432252]

Postle BR. Delay-period activity in the prefrontal cortex: one function is sensory gating. J Cogn Neurosci. 2005; 17(11):1679-1690.10.1162/089892905774589208 [PubMed: 16269105]

Ptito A, Crane J, Leonard G, Amsel R, Caramanos Z. Visual-spatial localization by patients with frontal-lobe lesions invading or sparing area 46. Neuroreport. 1995; 6(13):1781-1784. [PubMed: 8541481]

Raye CL, Mitchell KJ, Reeder JA, Greene EJ, Johnson MK. Refreshing one of several active representations: behavioral and functional magnetic resonance imaging differences between young and older adults. J Cogn Neurosci. 2008; 20(5):852-862.10.1162/jocn.2008.20508 [PubMed: 18201130]

Rissman J, Gazzaley A, D’Esposito M. Measuring functional connectivity during distinct stages of a cognitive task. Neuroimage. 2004; 23(2):752-763. [PubMed: 15488425]

Rottschy C, Langner R, Dogan I, Reetz K, Laird AR, Schulz JB, Eickhoff SB. Modelling neural correlates of working memory: a coordinate-based meta-analysis. Neuroimage. 2012; 60(1):830846.10.1016/j.neuroimage.2011.11.050 [PubMed: 22178808]

Rypma B, Berger JS, D'Esposito M. The influence of working-memory demand and subject performance on prefrontal cortical activity. J Cogn Neurosci. 2002; 14(5):721731.10.1162/08989290260138627 [PubMed: 12167257]

Rypma B, D'Esposito M. A subsequent-memory effect in dorsolateral prefrontal cortex. Brain Res Cogn Brain Res. 2003; 16(2):162-166. S0926641002002471. [PubMed: 12668223]

Sakai K, Rowe JB, Passingham RE. Active maintenance in prefrontal area 46 creates distractorresistant memory. Nat Neurosci. 2002; 5(5):479-484.10.1038/nn846 [PubMed: 11953754]

Sawaguchi T, Yamane I. Properties of delay-period neuronal activity in the monkey dorsolateral prefrontal cortex during a spatial delayed matching-to-sample task. J Neurophysiol. 1999; 82(5): 2070-2080. [PubMed: 10561388]

Shima K, Tanji J. Role for cingulate motor area cells in voluntary movement selection based on reward. Science. 1998; 282:1335-1338. [PubMed: 9812901] 
Smith EE, Geva A, Jonides J, Miller A, Reuter-Lorenz P, Koeppe RA. The neural basis of taskswitching in working memory: effects of performance and aging. Proc Natl Acad Sci U S A. 2001; 98(4):2095-2100.10.1073/pnas.98.4.2095 [PubMed: 11172081]

Sreenivasan KK, Jha AP. Selective attention supports working memory maintenance by modulating perceptual processing of distractors. J Cogn Neurosci. 2007; 19(1):32-41.10.1162/jocn. 2007.19.1.32 [PubMed: 17214561]

Stuss DT, Alexander MP. Is there a dysexecutive syndrome? Philosophical Transactions of the Royal Society B. 2007; 362:901-915.

Thompson-Schill SL, D’Esposito M, Aguirre GK, Farah MJ. Role of left inferior prefrontal cortex in retrieval of semantic knowledge: a reevaluation. Proc Natl Acad Sci U S A. 1997; 94(26):1479214797. [PubMed: 9405692]

Thompson-Schill SL, Jonides J, Marshuetz C, Smith EE, D’Esposito M, Kan IP, Swick D. Effects of frontal lobe damage on interference effects in working memory. Cogn Affect Behav Neurosci. 2002; 2(2):109-120. [PubMed: 12455679]

Treisman A. Preattentive processing in vision. Computer vision, graphics, and image processing. 1985; 31(2):156-177.

Tsushima Y, Sasaki Y, Watanabe T. Greater disruption due to failure of inhibitory control on an ambiguous distractor. Science. 2006; 314(5806):1786-1788.10.1126/science.1133197 [PubMed: 17170308]

van Snellenberg, JX.; Wager, TD. Cognitive and Motivational Functions of the Human Prefrontal Cortex. In: Christensen, A.; Goldberg, E.; Bougakov, D., editors. Luria's Legacy in the 21 st Century. Oxford: Oxford University Press; 2009. p. 30-61.

Wager, TD.; Barrett, LF.; Bliss-Moreau, E.; Lindquist, K.; Duncan, S.; Kober, H.; Mize, J. The Neuroimaging of Emotion. In: Lewis, M.; Haviland-Jones, JM.; Barrett, LF., editors. Handbook of Emotions. 3. New York: Guilford Press; 2008. p. 249-271.

Wager TD, Keller MC, Lacey SC, Jonides J. Increased sensitivity in neuroimaging analyses using robust regression. Neuroimage. 2005; 26(1):99-113.10.1016/j.neuroimage.2005.01.011 [PubMed: 15862210]

Wang K, Yu C, Xu L, Qin W, Li K, Jiang T. Offline memory reprocessing: involvement of the brain's default network in spontaneous thought processes. PLoS One. 2009; 4(3):e4867.10.1371/ journal.pone.0004867 [PubMed: 19290044]

Weissman DH, Roberts KC, Visscher KM, Woldorff MG. The neural bases of momentary lapses in attention. Nat Neurosci. 2006; 9(7):971-978.10.1038/nn1727 [PubMed: 16767087] 


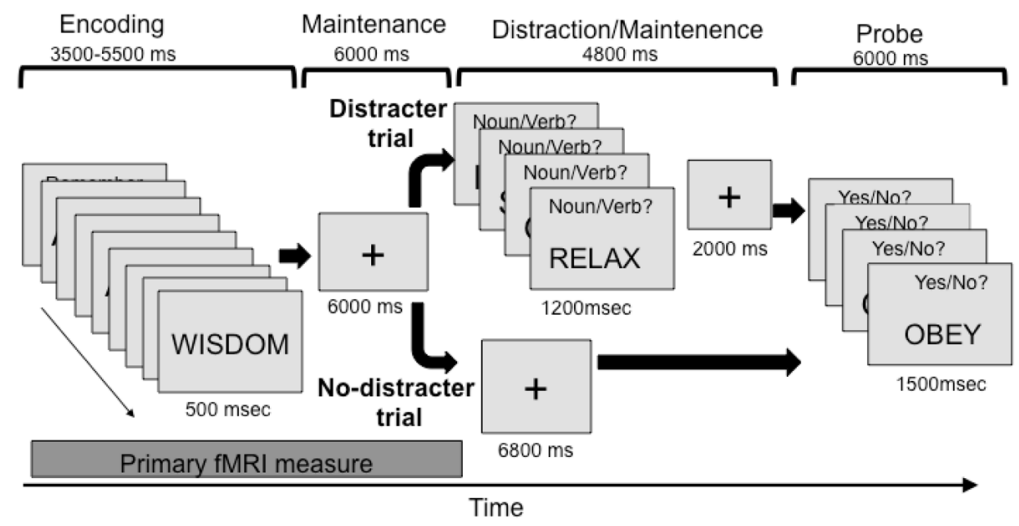

Figure 1.

Task design. During Encoding, memoranda were presented every $500 \mathrm{~ms}$, with the number of words for each participant determined before the main task using an adaptive procedure targeting 75\% accuracy. During Maintenance, participants rehearsed the memoranda. On Distracter trials, Maintenance was followed with a secondary task in which participants made noun/verb judgments on four words. On No-Distracter trials, participants continued maintaining the items for the same duration as the secondary task on Distracter trials (6800 $\mathrm{ms}$ ). In the Probe period, participants saw four words and made yes/no judgments as to whether they were part of the memory set. 


\section{A}

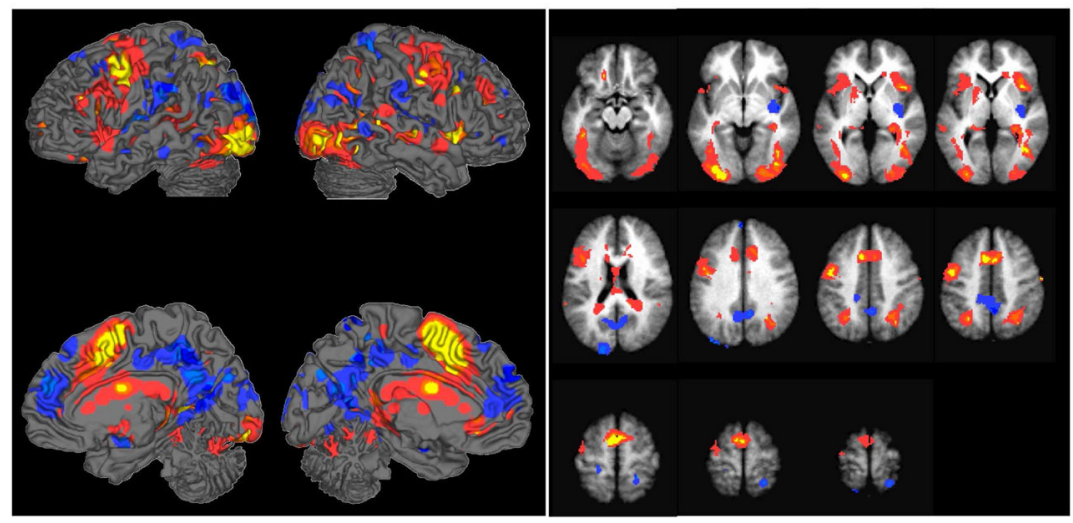

B
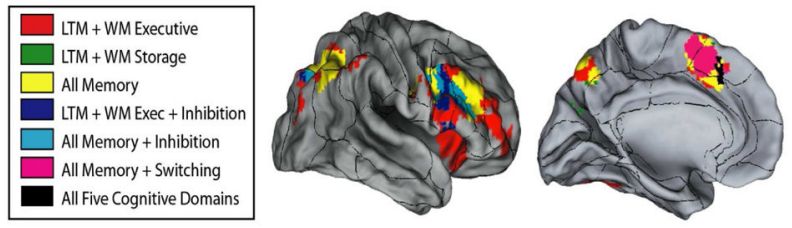

Figure 2.

FMRI activity related to working memory Encoding/Maintenance. A) Activations and deactivations in the [Encoding/Maintenance - Baseline] contrast, shown in red/yellow colors and purple/blue, respectively. The primary threshold was $\mathrm{p}<10^{-6}$ (yellow/dark blue for positive/negative effects) and 3 contiguous voxels. Contiguous voxels at lower thresholds that are contiguous with those at the primary threshold are also shown, at $\mathrm{p}<10^{-5}$ (orange/ light blue) and $\mathrm{p}<.009$ ( $\mathrm{q}<.01$ False Discovery Rate corrected, shown in pink/purple). This stringent threshold was chosen to provide anatomically specific regions of interest, though activity in this contrast was widespread. The anatomical underlay is the average T1weighted image from this sample, after normalization to Montreal Neurological Institute space. B) Results from meta-analyses of working memory (WM), long-term memory (LTM), task switching, and inhibition tasks for comparison with (A). WM is divided into executive (Exec) and storage-only tasks. The black lines show anatomical divisions between Brodmann's Areas as implemented in Caret software (http://brainvis.wustl.edu/wiki/ index.php/Caret:About). Figure adapted from van Snellenberg et al. (2009). 


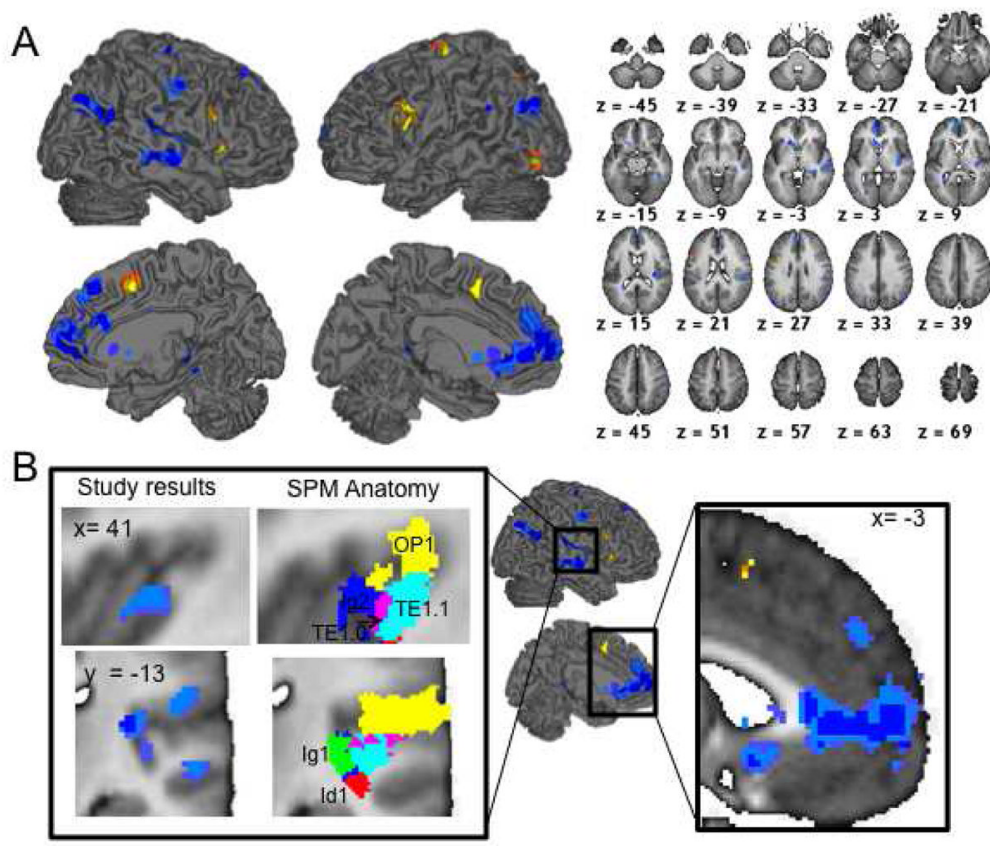

Figure 3.

Regions both responsive to working memory demand and predictive of subsequent accuracy. A) Surface rendering of areas significant in the conjunction of [Encoding/ Maintenance - Baseline] and subsequent accuracy. In yellow/orange areas, activity increases predicted higher accuracy, and in blue/purple areas, activity decreases predicted higher accuracy. Right: axial slices showing results for this conjunction. The threshold is $\mathrm{p}<.001$ (yellow/dark blue), $\mathrm{p}<.005$ (orange/light blue), and $\mathrm{p}<.01$ (pink/purple), with voxels at the latter thresholds contiguous with those at higher thresholds. B) Left: Detailed localization of sensory regions, compared with the anatomical regions from the SPM Anatomy Toolbox v. 1.7. Deactivations predicting accurate memory were localized in somatosensory areas OP1 (SII) and dorsal posterior insula (extending into Ig1) and auditory areas TE1.1/1.0. Right: Increases in pre-SMA and decreases in ventromedial prefrontal cortex predictive of accurate performance. The full extent of activation is shown for display purposes, although only the more dorsal portion was significant in the [Encoding/Maintenance - Baseline] contrast. 

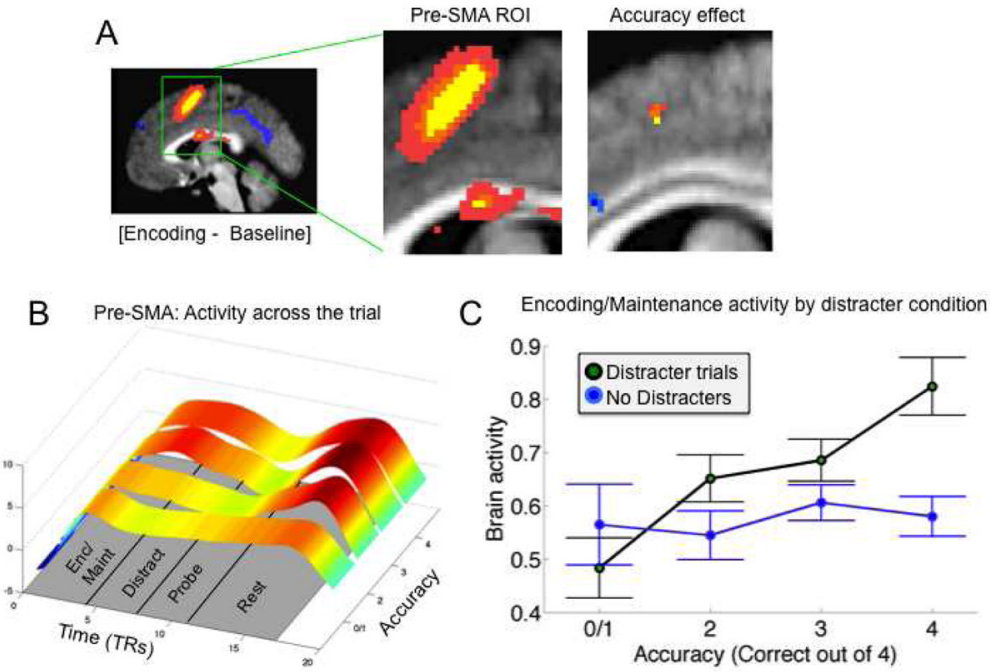

Figure 4.

Pre-supplementary motor area results. A) The significant region in used as a functional region of interest (ROI) for distraction analyses. Left, [Encoding/Maintenance - Baseline] results, with thresholds as in Figure 2. Right: Voxels within this area correlating with accuracy, with thresholds as in Figure 3, located in the inferior frontal gyrus. B) Average activity in the accuracy-predictive ROI across the entire trial, for each level of accuracy. During the critical Encoding/Maintenance period, activity on trials with the highest level of accuracy (3 or 4 correct) is higher than trials with lower levels of accuracy. High levels of activation were also found during the probe interval, which is typical for studies of WM and consistent with findings on retrieval-related frontal activation, but is not of primary interest here. C) Plot of activity in the critical Encoding/Maintenance period ( $y$-axis) as a function of accuracy ( $x$-axis) and Distracter status. The positive overall relationship between brain activity and accuracy is driven by the Distracter condition. 


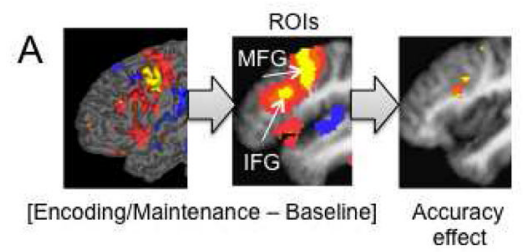

B Left IFG: Activity across the trial

C Encoding/Maintenance activity by distracter condition
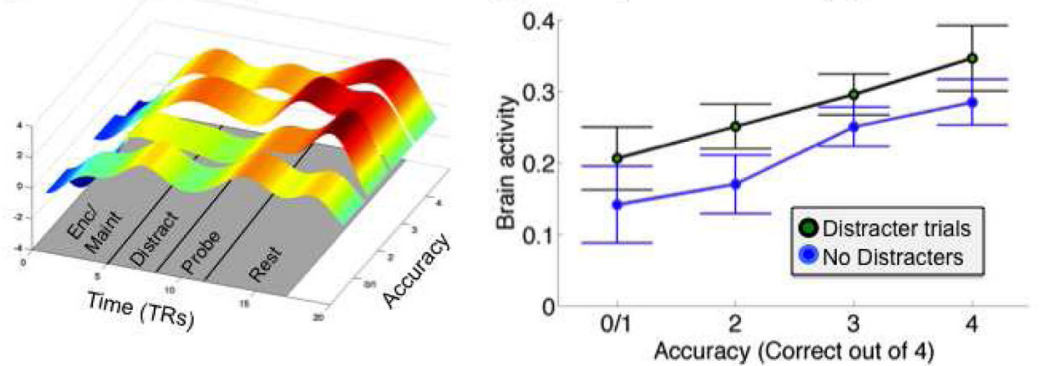

Figure 5.

Ventrolateral prefrontal cortex results. A) The significant region in used as a functional region of interest, as Figure 4. B) Average activity in the accuracy-predictive ROI across the entire trial, for each level of accuracy. C) Plot of activity as a function of accuracy and Distracter status. The relationship between brain activity and accuracy held for both Distracter and No-Distracter trials. 
Accuracy $\times$ Distraction Interaction: Frontal Regions

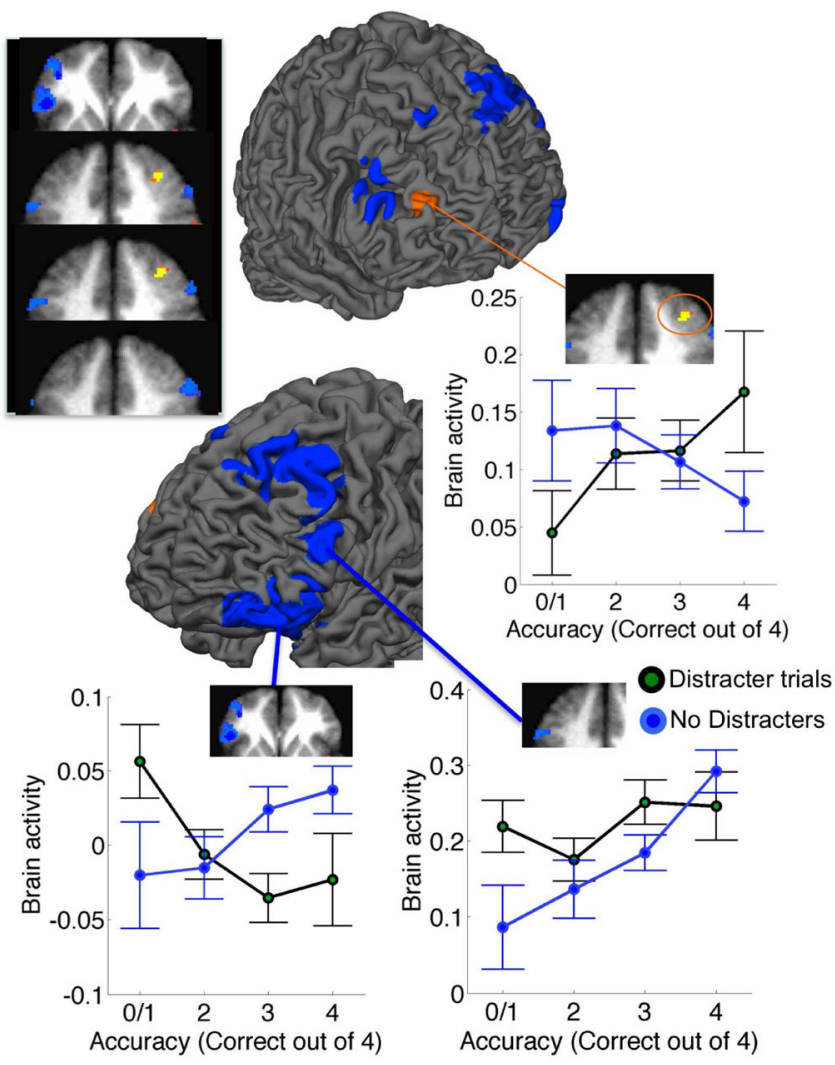

Figure 6.

Accuracy $x$ Distraction interaction results across the functional cortex. These results are not constrained to functional regions of interest. In yellow/orange areas, the activityperformance relationship was more positive for the Distracter trials. In blue areas, the relationship was more positive for the No-Distracter trials. Thresholds and colors are as in Figure 3. Whole-brain results for the Accuracy x Distraction interaction are shown in Supplementary Figure 1. 

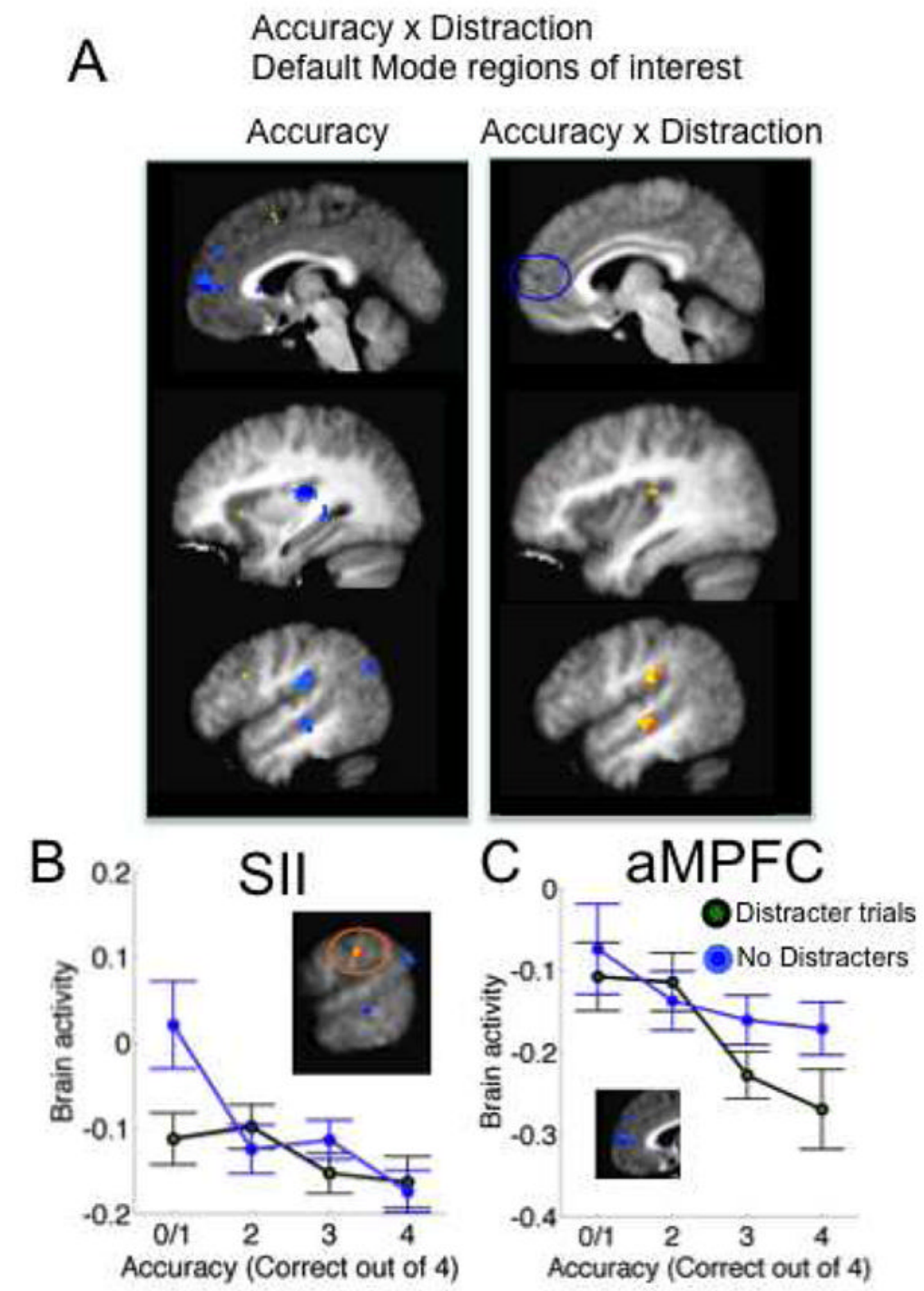

Figure 7.

Accuracy $x$ Distraction interaction results in default mode and sensory regions of interest. A) Left, regions predictive of accuracy and significantly activated during Encoding/ Maintenance. Right: Voxels showing Accuracy $x$ Distraction interactions. Thresholds and colors are as in Figure 3. In yellow/orange areas, the activity-performance relationship was more positive for the Distracter trials. B) Detail for secondary somatosensory cortex (SII). In sensory areas, including SII and dorsal-posterior insula, deactivation was more strongly related to performance in the No-distracter condition. C) In anterior medial prefrontal cortex (aMPFC), deactivation was more strongly related to performance in the Distracter condition. 


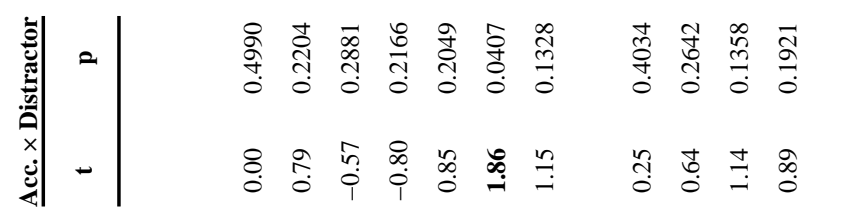

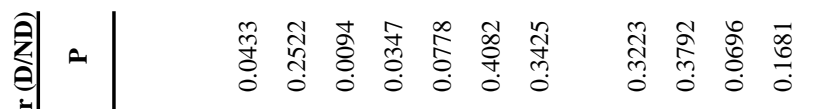

苗

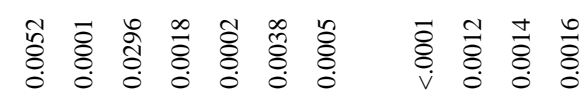

芛

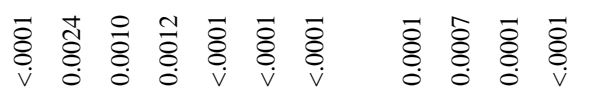

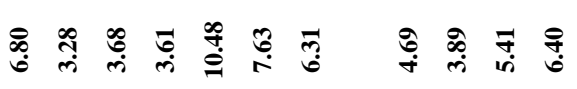

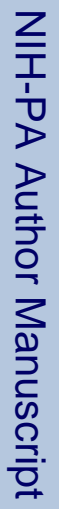

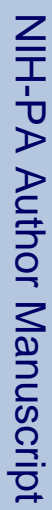

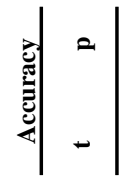

离

氞

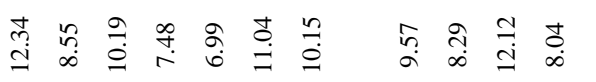

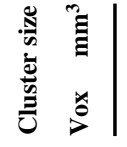

品

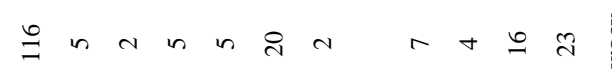

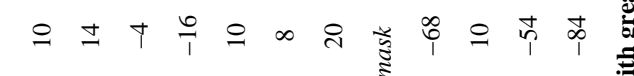

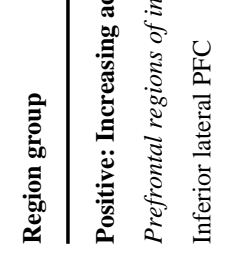

壱

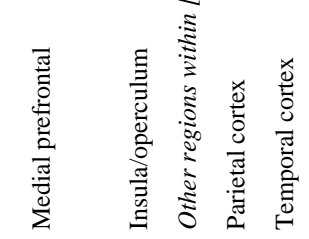

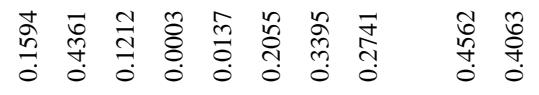

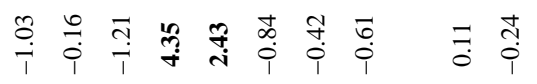

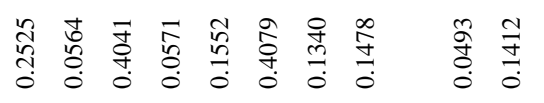

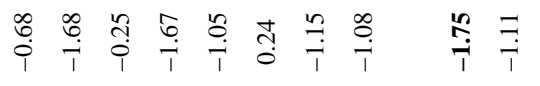

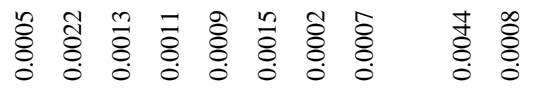

商

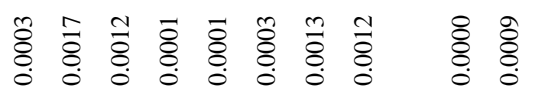

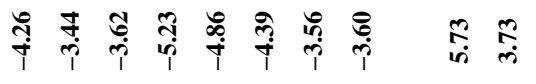

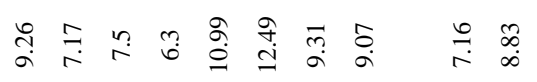

悹

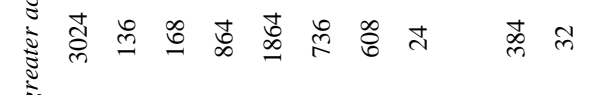

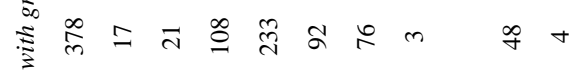

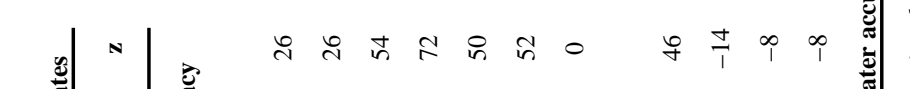

芯

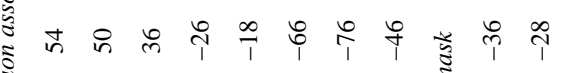

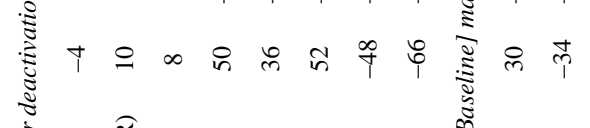

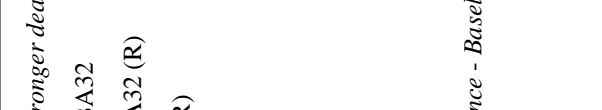

¿

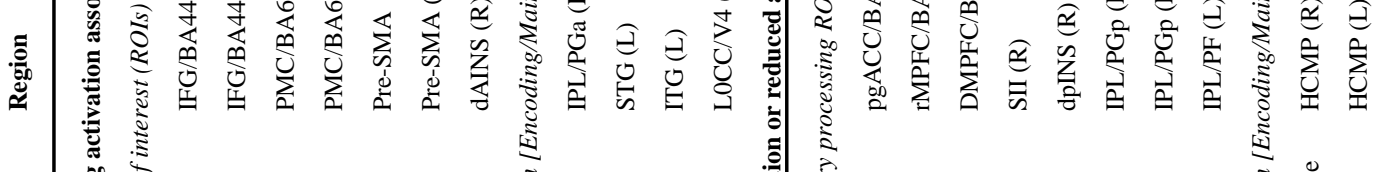

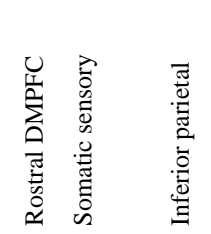

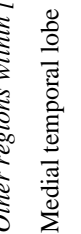




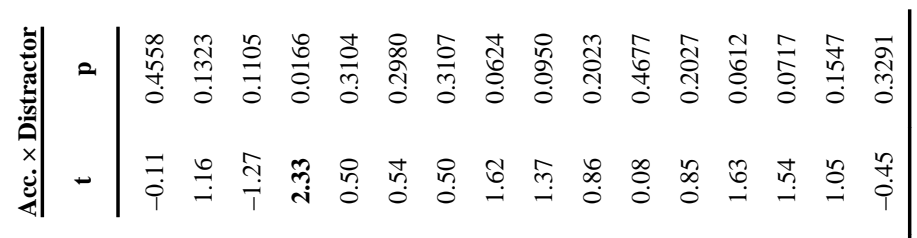

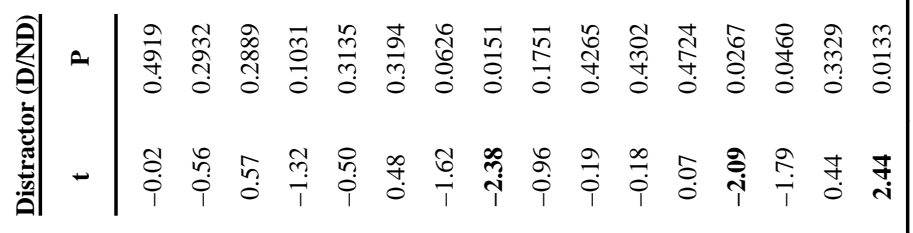

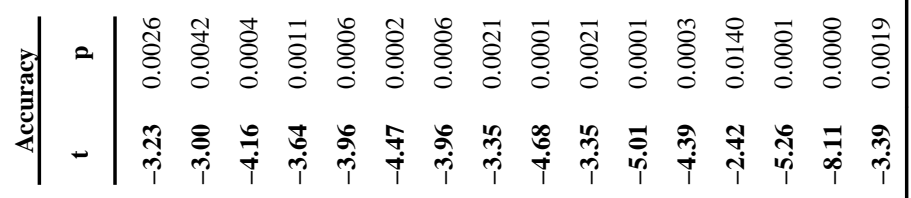

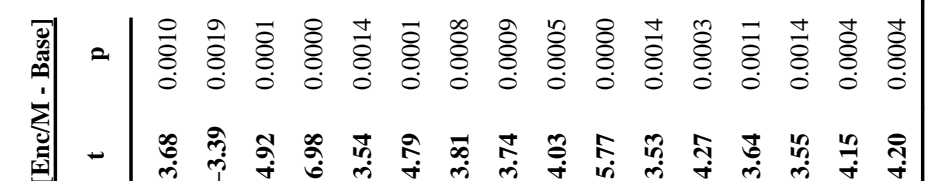

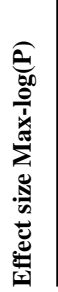

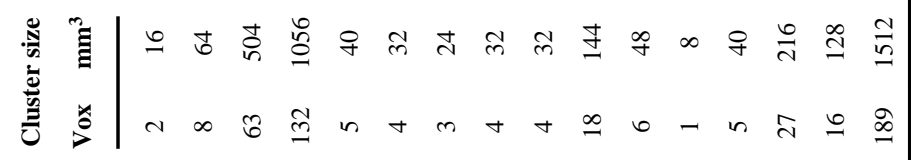

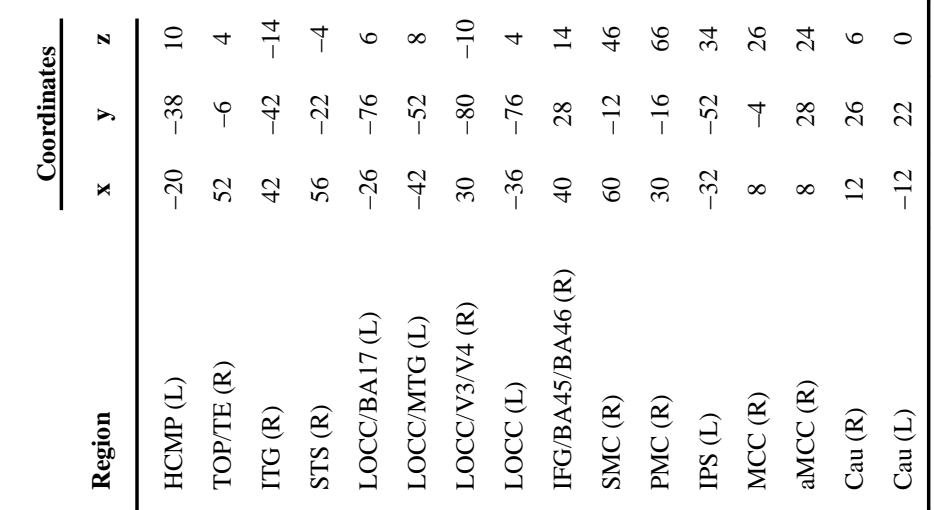

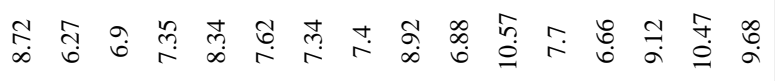

